\title{
Characterizing the Effect of Coarse-Scale PBN Modeling on Dynamics and Intervention Performance of Genetic Regulatory Networks Represented by Stochastic Master Equation Models
}

\author{
Ranadip Pal, Member, IEEE, and Sonal Bhattacharya
}

\begin{abstract}
Fine-scale models based on stochastic master equations can provide the most detailed description of the dynamics of gene expression and imbed, in principle, all the information about the biochemical reactions involved in gene interactions. However, there is limited time-series experimental data available for inference of such fine-scale models. Furthermore, the computational complexity involved in the design of optimal intervention strategies to favorably effect system dynamics for such detailed models is enormous. Thus, there is a need to design mappings from finescale models to coarse-scale models while maintaining sufficient structure for the problem at hand and to study the effect of intervention policies designed using coarse-scale models when applied to fine-scale models. In this paper, we propose a mapping from a fine-scale model represented by a stochastic master equation to a coarse-scale model represented by a probabilistic Boolean network that maintains the collapsed steady state probability distribution of the detailed model. We also derive bounds on the performance of the intervention strategy designed using the coarse-scale model when applied to the fine-scale model.
\end{abstract}

Index Terms-Markov chain models, probabilistic Boolean networks, relationships between genetic regulatory network models, stochastic master equations.

\section{INTRODUCTION}

$\mathbf{T}$ HE sequencing of various genomes and technological advances in gene expression measurement over the last decade has provided tremendous opportunities for mathematical modeling of gene regulatory networks. There are two major objectives for modeling of genetic regulatory networks: first, to better understand the intergene interactions and relationships on a systems-level, thereby facilitating the diagnosis of disease; and second, to design and analyze therapeutic intervention strategies for shifting the state of a diseased network from an undesirable one to a desirable one. The first objective falls within the scope of the field known as systems biology, while

Manuscript received September 23, 2009; accepted February 01, 2010. Date of publication February 22, 2010; date of current version May 14, 2010. The associate editor coordinating the review of this manuscript and approving it for publication was Dr. Z. Jane Wang. This research was supported by NSF Grant CCF0953366.

The authors are with the Electrical and Computer Engineering Department, Texas Tech University, Lubbock, TX 79409 USA (e-mail: ranadip.pal@ttu.edu; sonal.bhattacharya@ttu.edu).

Color versions of one or more of the figures in this paper are available online at http://ieeexplore.ieee.org.

Digital Object Identifier 10.1109/TSP.2010.2043970 the second objective falls within the scope of the field known as systems medicine. This paper deals with an issue arising in systems medicine.

The selection of a mathematical model to describe the dynamical behavior of a genetic regulatory network is dependent on the available data, estimation techniques, model complexity and specific purpose of constructing the model. From a systems biology point of view, the mathematical model must necessarily mimic the actual molecular biological interactions in as much detail as possible. A stochastic master equation model [1]-[3] can provide such detailed description of the dynamics of gene expression. However, the estimation of the parameters of the fine scale model requires larger data sets and preferably time series data. Furthermore, cell specific measurements instead of tissue averaged measurements are preferable for the inference of fine scale stochastic models. In the event of accurate inference of the parameters of the detailed model, the design of optimal intervention strategies for such a model possess a huge computational problem. From a systems medicine point of view, the network used for the purpose of design of intervention strategies can be a coarse representation of the biological phenomena occurring at the molecular level as long as it has the capability to faithfully capture the overall effects of intervention that are manifested at the phenotypic (observational) level. For intervention purposes, the effectiveness of drugs can usually be characterized by observing the expression levels of oncogenes, tumor suppressor genes and molecular markers of tumor progression represented by few discrete levels (mostly binary, ternary or quaternary). In one of our prior case studies [4], [5], we were able to approximate our goal of intervention by the coarse-scale objective of minimizing the steadystate probability of mRNA molecules of a specific molecular marker of tumor progression (WNT5A) being higher than some threshold. In this context, an intervention approach designed from a coarse-scale model and producing the desired behavior in the coarse-scale model will be reasonable if the designed policy when applied to the fine scale model also produces similar coarse-scale behavior.

The basic systems approach to cancer therapy can be described as a series of steps shown in Fig. 1. Intervention in genetic regulatory networks has been mainly studied to date using the coarse-scale probabilistic Boolean network model [6]. In 


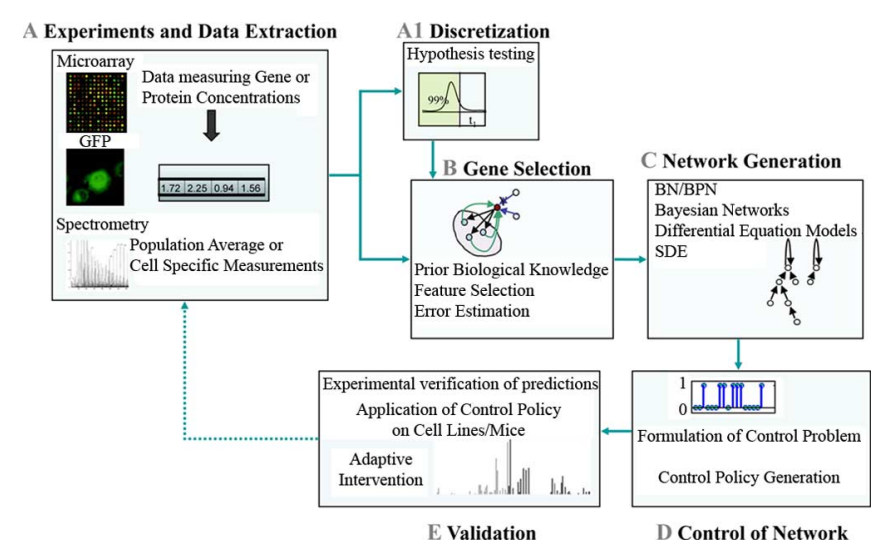

Fig. 1. Basic steps involved in modeling and control of genetic networks: A: Biological Experiments conducted to extract gene or protein expression data. $\left(\mathrm{A}_{1}\right)$ Discretization of the data (not required for continuous models). B: Selection of a small set of genes relevant to the pathway of interest. C: Generation of the network from the available data and prior biological knowledge. D: Intervention in the network with the objective of moving the network from undesirable to desirable states. E: Validation of the predictions arising from network analysis, validation of the intervention approach and closing the loop.

this paper, we will present a reduction mapping from a detailed model represented by a stochastic master equation (SME) to a coarse-scale model represented by a probabilistic Boolean network $(\mathrm{PBN})$ while maintaining the collapsed steady state probability distribution. We will also derive bounds on the effect of the control policy designed using the PBN when applied to the detailed model. The objective is to study the conditions under which the desired control behavior can be attained in the fine-scale model by application of a control policy designed using coarse-scale models. We are assuming that the actual genetic regulatory network can be represented by a fine-scale stochastic master equation model but we are only able to infer a coarse-scale model due to limitations on experimental data. Our goal is to study the dynamic properties maintained by the coarse-scale model as compared to the fine scale model and the actual performance of the control strategy designed using the coarse-scale model.

Fine-scale models based on stochastic master equations can provide the most detailed description of the dynamics of gene expression and imbed, in principle, all the information about the biochemical reactions involved in gene interactions. However, the use of fine-scale stochastic models is restricted by parameter inference problems due to inadequate time series data and technological limitations on cell specific measurements and the inherent computational complexity involved in its simulation [7]. Furthermore, the computational complexity involved in the design of optimal intervention strategies to favorably effect system dynamics for such detailed models is enormous. For instance, if we consider the standard dynamic programming approach to calculate the optimal control policy for a discrete time stochastic master equation model with $n$ genes and possible $k$ mRNA/protein molecules for each gene and $m$ binary control options, then each step of the Bellman recursion will involve a complexity of $O\left(2^{m} k^{n}\right)$ [8]. The complexity is enormous as is evident if we consider $m=1$ control input, $k=40$ maximum mRNA/Protein molecules generated per gene and $n=5$ genes, then $2^{m} k^{n}=204800000=204.8$ million. If we use a reduced PBN model with $k=2$, then the complexity becomes a manageable $2^{1} 2^{5}=64$ calculations at each stage of the Bellman recursion.

The paper is organized as follows. We provide a brief description of the SME and the PBN model in Section II. Review and mathematical description of intervention are provided in Section III. The reduction mapping is presented in Section IV. We derived bounds on the control performance on Section V. Section VI contains simulation examples. Conclusions are provided in Section VII.

\section{REVIEW OF GENETIC REGUlATORY NeTwORK MODELS}

\section{A. Stochastic Master Equation Models}

Given a set of genes/proteins, the evolution of their expression levels constitutes a dynamical system over time. A large number of approaches have been proposed to model the behavior of genetic regulatory networks, both deterministic and stochastic [7], [9], [10]. Nonlinear ordinary differential equations have been used in modeling molecular interactions [11]-[14]. Equations of the form of $d x_{1} / d t=k_{1} r-\gamma_{1} x_{1}$ are employed where $k_{1}$ and $\gamma_{1}$ are production and degradation constants respectively and $r$ is a regulation function dependent on other genes. Discrete abstractions of differential equations in the form of piecewise linear differential equations have also been proposed as models for gene regulatory networks [15], [16]. Differential equation (DE) models assume that species concentration vary continuously and deterministically which is questionable in case of gene regulation [17]-[22]. Thus, stochastic and discrete fine-scale models commonly known as SME models have been proposed for modeling genetic regulatory networks [1]-[3]. To explain the SME models for genetic regulatory networks, let us consider a system with $n$ molecular species and $m$ different reaction channels where the state of the system is defined by $\mathbf{x}=\left[\phi_{1}, \phi_{2}, \ldots, \phi_{n}\right], \mathbf{x} \in \mathbb{N}^{n}$ is a vector of integers representing a specific population of each of the $n$ molecular species. For such a system, given the probability density vector $p(\mathbf{x}, t)$ at time $t$, the probability that the system will be in the state $\mathbf{x}$ at time $t+d t$ is given by

$$
\begin{aligned}
p(\mathbf{x} ; t+d t)=p(\mathbf{x} ; t)( & \left.1-\sum_{\mu=1}^{m} a_{\mu}(\mathbf{x}) d t\right) \\
& +\sum_{\mu=1}^{m} p\left(\mathbf{x}-v_{\mu} ; t\right) a_{\mu}\left(\mathbf{x}-v_{\mu}\right) d t
\end{aligned}
$$

where $a_{\mu}(\mathbf{x}) d t$ denotes the probability that the $\mu$ th reaction will happen in a time step of length $d t$ and $v_{\mu}$ is the stoichiometric transition vector. From (1), the differential equation known as the chemical master equation (CME) [3] can be derived:

$$
\dot{p}(\mathbf{x} ; t)=-p(\mathbf{x} ; t) \sum_{\mu=1}^{m} a_{\mu}(\mathbf{x})+\sum_{\mu=1}^{m} p\left(\mathbf{x}-v_{\mu} ; t\right) a_{\mu}\left(\mathbf{x}-v_{\mu}\right) \text {. }
$$


By considering all the reactions beginning or ending at state $\mathbf{x}$, the time derivative of the probability density of state $\mathbf{x}$ can be written in vector form as [23]

$$
\dot{p}(\mathbf{x} ; t)=\left[p(\mathbf{x} ; t) \cdots p\left(\mathbf{x}-v_{m} ; t\right)\right]\left[\begin{array}{c}
-\sum_{\mu=1}^{m} a_{\mu}(\mathbf{x}) \\
\cdots \\
a_{m}\left(\mathbf{x}-v_{m}\right)
\end{array}\right] .
$$

As $n$ is finite, the number of different possible vectors for $\mathbf{x}$ is countable. Thus, we can map all the possible $\mathbf{X}$ 's $\in \mathbb{N}^{n}$ to a sequence $\mathbf{x}_{1}, \mathbf{x}_{2}, \ldots$ of elements in $\mathbb{N}$. Let us represent that enumeration as $\mathbf{X}:=\left[\mathbf{x}_{1}, \mathbf{x}_{2}, \ldots\right]$ and rewrite (3) as

$$
\dot{\mathbf{P}}(\mathbf{X} ; t)=\mathbf{P}(\mathbf{X} ; t) \mathbf{A}
$$

where $\mathbf{P}(\mathbf{X} ; t)=\left[p\left(\mathbf{x}_{1}, t\right), p\left(\mathbf{x}_{2}, t\right), \ldots\right]$ is the complete probability density state vector at time $t$ and $\mathbf{A}$ is the state reaction matrix. The matrix $\mathbf{A}$ has the following properties : it is independent of $t$, all of its diagonal elements are nonpositive, and all of its off-diagonal elements are non-negative and all the rows sum to exactly zero. For the case of finite number of reachable states, the solution can be computed as $\mathbf{P}(\mathbf{X} ; t)=\mathbf{P}(\mathbf{X}, 0) e^{\mathbf{A} t}[23]$.

\section{B. Probabilistic Boolean Network Models}

Discrete models with synchronous timing have been used since the late 1960s in the form of the Boolean network model [24]. In this model, gene expression is quantized to two levels: ON and OFF denoted by 1 and 0 , respectively. The expression level (state) of a gene is functionally related via a logical rule to the expression states of other genes. Specifically, a Boolean network (BN) is composed of a set $V=\left\{x_{1}, x_{2}, \ldots, x_{n}\right\}$ consisting of $n$ binary variables, each denoting a gene expression, and a set $F=\left\{f_{1}, f_{2}, \ldots, f_{n}\right\}$ of regulatory functions, such that for discrete time, $t=0,1,2, \ldots$, $x_{k}(t+1)=f_{k}\left(x_{1}(t), \ldots, x_{n}(t)\right)$. At any time point, the state of the network is given by an expression vector $\left(x_{1}(t), \ldots, x_{n}(t)\right)$, called the gene activity profile (GAP). In the Boolean model, the assumption of a single transition rule for each gene can be problematic with respect to inference. The data are typically noisy, the number of samples is small relative to the number of parameters to be estimated, inputs to the system are typically not controlled and there is fundamental stochasticity of the genes in their mechanisms of action. Thus, one may observe various possible transitions from a given state. A salient instance is that unobserved (latent) variables external to the model network result in the network structure taking on various realizations. Owing to these considerations, a stochastic rule-based regulatory model, the PBN was proposed [25]. A PBN is composed of a collection $\left\{B_{1}, B_{2}, \ldots, B_{r}\right\}$ of finite-state-space networks having a small probability of perturbation $p$ and a transition probability between the networks denoted by $q$. The probabilistic structure of the PBN can be modeled as a Markov chain. Relative to their Markovian structure, PBNs are related to Bayesian networks [26]; specifically, the transition probability structure of a PBN can be represented as a dynamic Bayesian network and every dynamic Bayesian network can be represented as a PBN (actually, a class of PBNs) [27]. The rule-based structure of PBNs mirrors the functional transcrip- tional regulation in cells and their stochastic structure allows them to reflect uncertainty, both in the data and model selection. In this paper, we will be concerned with the Markov Chain associated with the PBN.

\section{REVIEW OF INTERVENTION USING GENETIC REgUlatory NETWORKS}

A key objective of modeling genetic regulatory networks is to use them to design protocols for affecting network dynamics. To date, intervention studies have focused largely on BNs and PBNs. Recently, some approaches on intervention in genetic regulatory networks using ordinary differential models or stochastic differential equation models have been proposed but the intervention has been designed for a simplified model of very few genes [28]. We next provide a brief description of the several intervention approaches that have been designed to date for PBNs.

The motivation behind application of control theory is to devise optimal policies for manipulating control variables that affect the transition probabilities of the network and can, therefore, be used to desirably affect its dynamic evolution. This method depends on the Markov chain induced by the PBN and therefore is extendable to other dynamical systems based on graphical models, such as dynamic Bayesian networks. In practice, intervention will be achieved by a) targeted small molecule kinase inhibitors (Imatinib, Gefitinib, Erlotinib, Sunitinib, etc. [29]), b) Monoclonal antibodies altering the protein concentrations (Cetuximab, Alemtuzumab, Trastuzumab, etc. [29]), or c) gene knockdowns [30]. The state desirability is determined by the values of genes/proteins associated with phenotypes of interest. The initial approach consisted of favorably altering the mean first passage times (MFPTs), increasing MFPTs to undesirable states and decreasing MFPTs to desirable states, in the Markov chain associated with a PBN via a one-time perturbation of the expression of a control gene [31]. Shortly thereafter, stochastic control theory was used to alter the transient dynamics over a finite time horizon [32]. Subsequently, infinite-horizon control, with the goal of favorably altering the steady-state distribution of the network via a stationary control policy [4] was considered. To explain infinite-horizon intervention in PBNs, we next provide a brief mathematical description of the control problem. A PBN with control can be modeled as a finite state Markov chain [4], [33] described by the control-dependent one-step transition probability $p_{i j}(u):=P\left(z_{t+1}=j \mid z_{t}=\right.$ $i, u_{t}=u$ ) where, for all $t$, the state $z_{t}$ is an element of a space $S$ and the control input $u_{t}$ is an element of a space $C$. The states make transitions according to $\omega:=\left(P^{u}\right)_{u \in C}$. In this case, once a control input is chosen, the resulting controlled transition probability matrix is uniquely determined. Let $\mu=$ $\left(u_{1}, u_{2}, \ldots\right)$ represent a generic control policy and $\Pi$ represent the set of all possible $\mu$ 's, i.e., the set of all possible control policies. Let $J_{\mu, \omega}$ denote the expected total cost for the discounted cost infinite-horizon problem [4] under control policy $\mu$ and transitions $\omega$ :

$$
J_{\mu, \omega}\left(z_{0}\right)=\lim _{M \rightarrow \infty} E_{z_{t+1}}\left\{\sum_{t=0}^{M-1} \alpha^{t} \tilde{g}\left(z_{t}, u_{t}, z_{t+1}\right)\right\}
$$


where $0<\alpha<1$ denotes the discount factor and $\tilde{g}\left(z_{t}, u_{t}, z_{t+1}\right)$ represents the cost of going from state $z_{t}$ to $z_{t+1}$ under the control action $u_{t} . \tilde{g}$ is higher for undesirable destination states. For the same destination states, $\tilde{g}$ is higher when the control is active versus when it is not. In the general formulation, the inclusion of the discount factor $\alpha$ in the cost captures the fact that costs incurred at a later time are less significant. In the case of cancer treatment, it signifies that the condition of the patient in the initial stages of treatment is more important than the condition at a later stage, or in other words, the reward for improving the condition of the patient in the present is more significant than the reward obtained from similar improvement at a later stage. This approach is reasonable if we keep in mind the expected life span of the patient.

The control problem here corresponds to minimizing the cost in (5). Consequently, the optimal infinite-horizon discounted cost is given by

$$
\Phi\left(\Pi, \omega, z_{0}\right):=\min _{\mu \in \Pi} J_{\mu, \omega}\left(z_{0}\right) .
$$

We are mainly interested in infinite-horizon control because such policies allow us to optimally alter the steady-state distribution of the network by driving its probability mass into desirable states. In [4], we utilized a stationary control policy to shift the steady-state distribution for a PBN derived from gene expression data collected in a metastatic melanoma study.

\section{Reduction MAPPING AND Associated TRANSITION PROBABILITIES}

If we consider the number of possible $\mathbf{x}_{i}$ 's to be finite, then the exact solution to the CME can be obtained through the exponential equation $\mathbf{P}(\mathbf{X} ; t)=\mathbf{P}(\mathbf{X}, 0) e^{\mathbf{A} t}$. This kind of situation can arise in biological systems where the number of mRNA/Protein molecules that can be generated are bounded. If for systems, the dimension of $\mathbf{X}$ is infinite, we can apply the finite state projection approach [23] to arrive at a finite truncation of the state space. Henceforth, we will consider a CME with finite number of states $M$ and our purpose is to transform that model to a smaller scale PBN model while maintaining some characteristics of the CME model. As the steady state distribution of the genetic regulatory network is considered representative of the phenotype, our objective will be to maintain the steady state distribution. The finite state continuous time CME model can be approximated by a discrete-time sampled Markov chain by using a suitable time period $\Delta t$. The $M \times M$ state transition probabilities of the discrete-time Markov chain denoted by $P_{\Delta}$ is given by the equation $P_{\Delta}=e^{\mathbf{A} \Delta t}$. We will map these transition probabilities to a $N \times N$ transition probability matrix of a PBN denoted by $P_{r}$ where $N$ will be $2^{n}$ for binary levels and $3^{n}$ for the case of ternary levels. We will consider binary PBNs from now onwards but the derivation for more than two levels will be similar. Let $T_{i}$ for $i \in\{1,2, \ldots n\}$ denote the thresholds for binarization. For instance, if $\mathbf{x}(t)=\left[\Psi_{1}, \Psi_{2}, \ldots \Psi_{n}\right]$, then the corresponding state for the PBN is $\mathbf{y}=\left[\psi_{1}, \psi_{2}, \ldots \psi_{n}\right]$ where $\psi_{i}=1$ if $\Psi_{i} \geq T_{i}$ or $\psi_{i}=0$ if $\Psi_{i}<T_{i}$ for all $i \in\{1,2, \ldots n\}$. As the states of the PBN will be $n$-dimensional vectors ranging from $00 \ldots 0$ to $11 \ldots 1$, we can represent them by the decimal equivalent state $z_{t}$ where $z_{t}$ ranges from 1 to $2^{n}$. Let us consider a sequence $0=a_{0}, a_{1}, \ldots, a_{N}=M$ such that $a_{i}-a_{i-1}$ for $i \in\left\{1,2, \ldots, 2^{n}\right\}$ denote the number of states in the CME model that map to state $i$ in the PBN model. The states of the CME model will be denoted by $w_{t}$ ranging from 1 to $M$. The states will be re-ordered such that the states $a_{i-1}+1$ to state $a_{i}$ of the CME will be mapped to state $i$ of the PBN for $i=1, \ldots, N$. Note that the subscript $\Delta$ refers to the higher dimensional discrete time CME model whereas the subscript $r$ refers to the lower dimensional PBN model.

Let the state of the PBN at time $t$ be $z_{t}=i$ and the state at time $t+1$ be $z_{t+1}=j$. The transition probability of going from state $i$ to state $j$ is given by [34]

$$
\begin{aligned}
& P_{r}(i, j) \\
& =P\left(z_{t+1}=j \mid z_{t}=i\right) \\
& =\sum_{j_{1}=a_{j-1}+1}^{a_{j}} P\left(w_{t+1}=j_{1} \mid z_{t}=i\right) \\
& =\frac{\sum_{j_{1}=a_{j-1}+1}^{a_{j}} P\left(w_{t+1}=j_{1}, z_{t}=i\right)}{P\left(z_{t}=i\right)} \\
& =\frac{\sum_{j_{1}=a_{j-1}+1}^{a_{j}} \sum_{i_{1}=a_{i-1}+1}^{a_{i}} P\left(w_{t+1}=j_{1}, w_{t}=i_{1}\right)}{\sum_{i_{2}=a_{i-1}+1}^{a_{i}} P\left(w_{t}=i_{2}\right)} \\
& =\frac{\sum_{j_{1}=a_{j-1}+1}^{a_{j}} \sum_{i_{1}=a_{i-1}+1}^{a_{i}} P\left(w_{t+1}=j_{1} \mid w_{t}=i_{1}\right) P\left(w_{t}=i_{1}\right)}{\sum_{i_{2}=a_{i-1}+1}^{a_{i}} P\left(w_{t}=i_{2}\right)} \\
& =\frac{\sum_{j_{1}=a_{j-1}+1}^{a_{j}} \sum_{i_{1}=a_{i-1}+1}^{a_{i}} P_{\Delta}\left(i_{1}, j_{1}\right) P\left(w_{t}=i_{1}\right)}{\sum_{i_{2}=a_{i-1}+1}^{a_{i}} P\left(w_{t}=i_{2}\right)} .
\end{aligned}
$$

We next show that if we use the steady state probabilities of the CME model for $P\left(w_{t}=i_{1}\right)$ in (7), then the steady state probabilities of the resultant PBN, $P_{r}$, will be the same as the collapsed steady state probabilities of the CME model. Here collapsing refers to the summation of the state probabilities of the detailed model that map to a certain state of the PBN model. For instance, if $\eta$ represents the $M$ dimensional steady state probability vector for the CME model, then the collapsed steady state vector will be a $N$ dimensional vector represented by $\zeta$ where $\zeta(i)=\sum_{c=a_{i-1}+1}^{a_{i}} \eta(c)$ for $i=1 \ldots N$. We next prove the equivalence of $\zeta$ and the steady state probabilities of the PBN represented by the following state transition probabilities:

$$
P_{r}(i, j)=\frac{\sum_{j_{1}=a_{j-1}+1}^{a_{j}} \sum_{i_{1}=a_{i-1}+1}^{a_{i}} P_{\Delta}\left(i_{1}, j_{1}\right) \eta\left(i_{1}\right)}{\sum_{i_{2}=a_{i-1}+1}^{a_{i}} \eta\left(i_{2}\right)} .
$$


Theorem IV.1: With (8) denoting the transition probabilities of the PBN, the steady state probability distribution vector, $\pi$, of the PBN is given by

$$
\pi(i)=\sum_{i_{2}=a_{i-1}+1}^{a_{i}} \eta\left(i_{2}\right)=\zeta(i)
$$

for $i=1, \ldots, N$.

Proof: Assuming an ergodic Markov Chain, $\zeta$ is the steady state distribution of the PBN if it satisfies the equation $\sum_{i=1}^{N} \zeta(i) P_{r}(i, j)=\zeta(j)$ for each $j \in 1,2 \ldots N$.

Since $\eta$ is the steady state distribution for the $M \times M \mathrm{CME}$ model $P_{\Delta}$, it satisfies $\eta\left(j_{1}\right)=\sum_{i_{0}=1}^{M} \eta\left(i_{0}\right) P_{\Delta}\left(i_{0}, j_{1}\right)$. Thus

$$
\begin{aligned}
\zeta(j) & =\sum_{j_{1}=a_{j-1}+1}^{a_{j}} \eta\left(j_{1}\right) \\
& =\sum_{j_{1}=a_{j-1}+1}^{a_{j}} \sum_{i_{0}=1}^{M} \eta\left(i_{0}\right) P_{\Delta}\left(i_{0}, j_{1}\right) \\
& =\sum_{j_{1}=a_{j-1}+1}^{a_{j}} \sum_{i=1}^{N} \sum_{i_{1}=a_{i-1}+1}^{a_{i}} \eta\left(i_{1}\right) P_{\Delta}\left(i_{1}, j_{1}\right) \\
& =\sum_{i=1}^{N} \sum_{i_{2}=a_{i-1}+1}^{a_{i}} \eta\left(i_{2}\right) P_{r}(i, j) \quad \text { (from eq . (8)) } \\
& =\sum_{i=1}^{N} P_{r}(i, j) \zeta(i) .
\end{aligned}
$$

This shows that $\zeta$ satisfies the steady state probability equations of the PBN and is equal to $\pi$ assuming an ergodic Markov Chain.

We have established that if the transition probabilities of the PBN are decided based on (8), then the steady state probabilities of the generated PBN is same as the collapsed steady state probabilities of the original detailed network. Thus, we are able to maintain the collapsed steady-state probabilities which might be indicative of a phenotype. If we revisit (8), we notice that $P_{r}(i, j)$ can be calculated from the transitions of the network once it has reached the steady state. If we observe the steady-state transitions of the genetic regulatory network modeled by the CME for a long time and generate the state-transition probabilities of the PBN $P_{r}(i, j)$ based on the number of transitions occurring from state $i$ to state $j$ divided by the total number of transitions from state $i$, we will arrive at (8). Thus, the mapping described by (8) exists between a network represented by a CME model and a coarse-scale PBN model when the transition probabilities of the PBN are inferred based on state transition data at steady-state.

\section{EFFECT ON INTERVENTION PERFORMANCE}

Let us consider the case that we have two control actions at each time step and $P_{\Delta_{1}}$ and $P_{\Delta_{2}}$ represent the stochastic master equation (chemical master equation) models corresponding to the two control actions. In practice, $P_{\Delta_{1}}$ might represent the model of the network with no drug delivery and $P_{\Delta_{2}}$ represent the model of the network following a drug delivery. Let, $P_{r_{1}}$ and $P_{r_{2}}$ represent the transition probability matrices of the PBNs generated from models $P_{\Delta_{1}}$ and $P_{\Delta_{2}}$ respectively using (8).
Since, we will design the control policy based on the reduced model, our control design will be based on $P_{r_{1}}$ and $P_{r_{2}}$. Let the goal of our control action be to alter the steady state probability distribution of the network and we design a stationary control policy using dynamic programming approaches based on $P_{r_{1}}$ and $P_{r_{2}}$ by minimizing (6). A stationary control policy for the case of two control actions for the PBN will be a binary vector of length $N$ where 0 at location $i$ denotes no control action when at state $i$ (the model of the network for state $i$ will then be based on $P_{r_{1}}$ ) whereas a 1 at location $i$ denotes control action when at state $i$ (the model of the network for state $i$ will then be based on $\left.P_{r_{2}}\right)$. To illustrate it further, let us consider a simple example where $N=2$,

$$
\begin{aligned}
P_{r_{1}} & =\left|\begin{array}{cc}
.2 & .8 \\
.65 & .35
\end{array}\right| \\
P_{r_{2}} & =\left|\begin{array}{ll}
.45 & .55 \\
.25 & .75
\end{array}\right|
\end{aligned}
$$

and stationary control policy $=\left[\begin{array}{ll}0 & 1\end{array}\right]$. Let $P_{r_{d}}$ denote the controlled PBN transition probability matrix for a specific stationary control policy. Thus, for the stationary control policy of [0 1], $P_{r_{d}}$ will have its first row from $P_{r_{1}}$ and second row from $P_{r_{2}}$ i.e.,

$$
P_{r_{d}}=\left|\begin{array}{cc}
.2 & .8 \\
.25 & .75
\end{array}\right|
$$

Thus, we will represent the designed controlled model as $P_{r_{d}}=T P_{r_{1}}+\left(I_{N}-T\right) P_{r_{2}}$ where $I_{N}$ represent the identity matrix of size $N \times N$ and $T$ is a $N \times N$ matrix with all entries zero except the diagonal entries corresponding to no control action equal to 1 . For the example earlier,

$$
T=\left|\begin{array}{ll}
1 & 0 \\
0 & 0
\end{array}\right|
$$

When a control policy designed using the PBN is applied to the fine-scale stochastic network model, the control policy corresponding to states $a_{i-1}+1, \ldots, a_{i}$ of the stochastic master equation model will be the same as the control policy for state $i$ of the PBN. Thus, the controlled fine scale model will be $P_{\Delta_{c}}=T_{\Delta} P_{\Delta_{1}}+\left(I_{M}-T_{\Delta}\right) P_{\Delta_{2}}$ where $T_{\Delta}$ is a $M \times M$ matrix of all zeros except the states that map to PBN states with no control action, equal to 1 . For the example before, if we consider $M=4$ and the states 1 and 2 of the fine-scale model map to state 1 of the PBN, then

$$
T_{\Delta}=\left|\begin{array}{llll}
1 & 0 & 0 & 0 \\
0 & 1 & 0 & 0 \\
0 & 0 & 0 & 0 \\
0 & 0 & 0 & 0
\end{array}\right| .
$$

Let $P_{r_{c}}$ represent the $N \times N$ size transition probability matrix of the PBN generated from $P_{\Delta_{c}}$ according to (8). Let $\pi_{d}$ and $\pi_{c}$ represent the steady-state probability distribution vectors for $P_{r_{d}}$ and $P_{r_{c}}$ respectively. Let $\eta_{c}$ be the length $M$ steady state probability distribution vector of $P_{\Delta_{c}}$ and $\zeta_{c}$ be the length $N$ collapsed steady state probability distribution vector of $P_{\Delta_{c}}$. Due to Theorem IV.1, $\pi_{c}$ is equal to $\zeta_{c}$.

Our control objective is to alter the steady state probabilities of the network which in our control design is represented 
by $\pi_{d}$. When the designed control policy is applied to the actual network represented by the fine-scale model, the resulting coarse-scale steady state probability vector is $\pi_{c}$. We would like to study the conditions for $\pi_{c}$ to be close to $\pi_{d}$ so that our control designed on a reduced network when applied to the fine-scale network will have similar coarse-scale performance.

Let

$$
u(i)= \begin{cases}1 & \text { if } T(i, i)=1 \\ 2 & \text { if } T(i, i)=0\end{cases}
$$

Then,

$$
\begin{aligned}
& P_{r_{c}}(i, j)=\frac{\sum_{j_{1}=a_{j-1}+1}^{a_{j}} \sum_{i_{1}=a_{i-1}+1}^{a_{i}} P_{\Delta_{u(i)}}\left(i_{1}, j_{1}\right) \eta_{c}\left(i_{1}\right)}{\sum_{i_{2}=a_{i-1}+1}^{a_{i}} \eta_{c}\left(i_{2}\right)} \\
&= \sum_{\sum_{1}=a_{j-1}+1}^{a_{j}} P_{i_{1}}=a_{i-1}+1 \\
& \zeta_{c}(i)
\end{aligned}
$$

and

$$
\begin{aligned}
P_{r_{d}}(i, j) & =\frac{\sum_{j_{1}=a_{j-1}+1}^{a_{j}} \sum_{i_{1}=a_{i-1}+1}^{a_{i}} P_{\Delta_{u(i)}}\left(i_{1}, j_{1}\right) \eta_{u(i)}\left(i_{1}\right)}{\sum_{i_{2}=a_{i-1}+1}^{a_{i}} \eta_{u(i)}\left(i_{2}\right)} \\
& =\frac{\sum_{j_{1}=a_{j-1}+1}^{a_{j}} \sum_{i_{1}=a_{i-1}+1}^{a_{i}} P_{\Delta_{u(i)}}\left(i_{1}, j_{1}\right) \eta_{u(i)}\left(i_{1}\right)}{\zeta_{u(i)}(i)}
\end{aligned}
$$

Let $\beta_{1}\left(i_{1}, j\right)=\sum_{j_{1}=a_{j-1}+1}^{a_{j}} P_{\Delta_{1}}\left(i_{1}, j_{1}\right)$ and $\beta_{2}\left(i_{1}, j\right)=$ $\sum_{j_{1}=a_{j-1}+1}^{a_{j}} P_{\Delta_{2}}\left(i_{1}, j_{1}\right)$.

Let us represent $\beta_{1}\left(i_{1}, j\right)$ and $\beta_{2}\left(i_{1}, j\right)$ for $i_{1} \in\left[a_{i-1}+\right.$ $\left.1, a_{i-1}+2, \ldots, a_{i}\right]$ as summation of two terms with the first one independent of $i_{1}$ and dependent on $i$ and $j$ and the second term dependent on $i_{1}$ and $j$.

$$
\begin{aligned}
& \beta_{1}\left(i_{1}, j\right)=t_{1}(i, j)+\epsilon_{1}\left(i_{1}, j\right) \text { and } \\
& \beta_{2}\left(i_{1}, j\right)=t_{2}(i, j)+\epsilon_{2}\left(i_{1}, j\right) .
\end{aligned}
$$

Let $\epsilon$ denote the maximum of $\left|\epsilon_{1}\left(i_{1}, j\right)\right|$ and $\left|\epsilon_{2}\left(i_{1}, j\right)\right|$ i.e., $-\epsilon \leq \epsilon_{1}\left(i_{1}, j\right) \leq \epsilon$ and $-\epsilon \leq \epsilon_{2}\left(i_{1}, j\right) \leq \epsilon \forall i_{1} \epsilon$ $[1,2, \ldots \bar{M}], j \in[1,2, \ldots N]$. The value of $\epsilon$ can be calculated as (13), shown at the bottom of the page.

The following theorem bounds the difference between the transition probabilities of $P_{r_{c}}$ and $P_{r_{d}}$ in terms of $\epsilon$.

Theorem V.1: $\left|P_{r_{c}}(i, j)-P_{r_{d}}(i, j)\right| \leq 2 \epsilon$ for $\forall i, j \in$ $[1,2, \ldots, N]$.

Proof: See (14)-(16), shown at the bottom of the next page.
A low value of $\epsilon$ signifies that the probabilities of leaving the state $i$ from the states mapping to state $i$ (i.e., $i_{1} \cdots i_{g}$ ) are very similar. $\epsilon$ can have a low value for cases such as with bimodal gene expressions where thresholds are selected to divide the distinct modes. We should also note that $\epsilon$ calculated by (14) can be large due to limited instances of largely different $\beta\left(i_{1}, j\right)$ 's among $i_{1} \in\left[a_{i-1}+1, \ldots, a_{i}\right]$. In other words, for an $i$ and $j$, $\operatorname{most} \beta\left(i_{1}, j\right)$ for $i_{1} \in\left[a_{i-1}+1, \ldots, a_{i}\right]$ can be similar but $\epsilon$ can be large due to the few largely different $\beta\left(i_{1}, j\right)$ 's. We should note here that since $a_{i}-a_{i-1}$ can be huge, the few $\left(\ll a_{i}-a_{i-1}\right)$ different $\beta\left(i_{1}, j\right)$ can unnecessarily produce a weak bound for $\left|P_{r_{c}}(i, j)-P_{r_{d}}(i, j)\right|$ using (16). To illustrate it further, let us consider $L=a_{i}-a_{i-1}$ and $\left|\epsilon_{u(i)}\left(i_{1}, j\right)\right|<\tau$ is valid for $L_{1}<L$ of $i_{1}$ 's and $\left|\epsilon_{u(i)}\left(i_{1}, j\right)\right|\langle\theta$ where $\theta \gg \tau$ is valid for the rest $L-L_{1} i_{1}$ 's. For this case, using (13) will produce $\epsilon \geq \theta$ and thus Theorem V.1 will produce an upper-bound of at least $2 \theta$ for $\left|P_{r_{c}}(i, j)-P_{r_{d}}(i, j)\right|$. We will show next that the upper bound for the expected value of $\left|P_{r_{c}}(i, j)-P_{r_{d}}(i, j)\right|$ will be closer to $2 \tau$ which will be a much tighter bound as compared to $2 \theta$. Without loss of generality, let us assume that $\left|\epsilon_{u(i)}\left(i_{1}, j\right)\right|<\tau$ is valid for $i_{1}=a_{i-1}+1, \ldots, a_{i-1}+L_{1}$ whereas $\left|\epsilon_{u(i)}\left(i_{1}, j\right)\right|<\theta$ is valid for $i_{1}=a_{i-1}+L_{1}+1, \ldots, a_{i}$. Then assuming independence of $\epsilon_{u(i)}\left(i_{1}, j\right)$ and $\eta_{c}\left(i_{1}\right)$, we have from (15)

$$
\begin{aligned}
& E_{i_{1}}\left(\left|\frac{\left.\sum_{i_{1}=a_{i-1}+1}^{a_{i}} \epsilon_{u(i)}\left(i_{1}, j\right) \eta_{c}\left(i_{1}\right) \mid\right)}{\zeta_{c}(i)}\right|\right) \\
& \leq \frac{\sum_{i_{1}=a_{i-1}+1}^{a_{i}} E_{i_{1}}\left|\epsilon_{u(i)}\left(i_{1}, j\right)\right| E_{i_{1}}\left|\eta_{c}\left(i_{1}\right)\right|}{\zeta_{c}(i)} \\
& \leq \frac{\sum_{i_{1}=a_{i-1}+1}^{a_{i}} E_{i_{1}}\left|\epsilon_{u(i)}\left(i_{1}, j\right)\right|}{L}\left(\text { as } E_{i_{1}}\left|\eta_{c}\left(i_{1}\right)\right|=\frac{\zeta_{c}(i)}{L}\right) \\
& \leq \frac{L_{1} \tau+\left(L-L_{1}\right) \theta}{L} .
\end{aligned}
$$

For $L-L_{1} \ll L, L_{1} \tau+\left(L-L_{1}\right) \theta / L$ will be very close to $\tau$ and thus the upper bound for $\left|P_{r_{c}}(i, j)-P_{r_{d}}(i, j)\right|$ will be closer to $2 \tau$.

Based on Theorem V.1, we can study the difference between the actual steady state distribution $\pi_{c}=\zeta_{c}$ after application of stationary control policy $T$ and the simulated steady state probability distribution $\pi_{d}$ based on the reduced PBN model.

The steady-state probability distribution $\pi_{c}$ of $P_{r_{c}}$ is the solution to the following linear equation:

$$
x\left(P_{r_{c}}-I_{N}\right)=\mathbf{0}
$$

$$
\epsilon=\max _{i, j \in[1, \ldots, N], u \in[1,2]} \frac{\left(\max _{i_{1} \in\left[a_{i-1}+1, \ldots, a_{i}\right]} \beta_{u}\left(i_{1}, j\right)-\min _{i_{1} \in\left[a_{i-1}+1, \ldots, a_{i}\right]} \beta_{u}\left(i_{1}, j\right)\right)}{2} .
$$


where $I_{N}$ is the identity matrix of size $N \times N$ and the solution vector $x$ is equal to $\pi_{c}$. The residual for the approximate solution $\pi_{d}$ is given by $\kappa=\pi_{d}\left(P_{r_{c}}-I_{N}\right)$ where the norm of $\kappa$ provides a measure of closeness of the approximate solution as compared to the true solution [35].

$$
\begin{aligned}
|\kappa|_{\infty} & =\left|\pi_{d}\left(P_{r_{c}}-I_{N}\right)\right|_{\infty} \\
& =\left|\pi_{d}\left(P_{r_{c}}-P_{r_{d}}\right)\right|_{\infty}\left(\operatorname{as} \pi_{d} I_{N}=\pi_{d}=\pi_{d} P_{d}\right) \\
& \leq 2 \epsilon(\text { from Theorem V.1). }
\end{aligned}
$$

Another way, we can bound the difference is based on perturbation bounds. For two Markov Chains with transition probabilities $P_{d}$ and $P_{r}$ and sharing a common state space, the difference between the two stationary distributions can be bounded by $\left|\pi_{d}-\pi_{c}\right|_{q} \leq k\|E\|_{\infty}$ where $q=1$ or $\infty$ and $k>0$ are some constants and $\left|\pi_{d}-\pi_{c}\right|_{q}$ refers to the $q$ th norm of the vector $\pi_{d}-\pi_{c}$ and $\|E\|_{\infty}$ denotes the $\infty$ norm of the error matrix $E=P_{d}-P_{r}$ which is equivalent to the maximum absolute row sum of $E$. The constants $k$ are usually referred to as condition numbers and several of them have been studied in the literature. Obviously, some of the condition numbers will yield tighter bounds than the others and [36] gives a nice comparison of the available bounds.

These perturbation bounds are mostly stated in terms of the fundamental matrix or the group inverse of $A:=I-P$. The fundamental matrix of the Markov Chain with transition probability matrix $P$ is defined by

$$
Z=\left(A+e \pi^{T}\right)^{-1}
$$

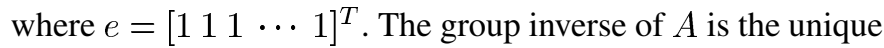
square matrix $A^{\#}$ satisfying the relationships

$$
A A^{\#} A=A, A^{\#} A A^{\#}=A^{\#}, \text { and } A A^{\#}=A^{\#} A .
$$

The currently available condition numbers for bounding the 1 and $\infty$ norms of the perturbations in the steady-state distributions are [36]

$$
\begin{aligned}
& k_{1}=\|Z\|_{\infty} q=1 \\
& k_{2}=\left\|A^{\#}\right\|_{\infty} q=1 \\
& k_{3}=\frac{\max _{j}\left(a_{j j}^{\#}-\min _{i} a_{i j}^{\#}\right)}{2} q=\infty \\
& k_{4}=\max _{i, j}\left|a_{i j}^{\#}\right| q=\infty \\
& k_{5}=\frac{1}{1-\tau_{1}(P)} q=1 \\
& k_{6}=\tau_{1}\left(A^{\#}\right)=\tau_{1}(Z) q=1 \\
& k_{7}=\frac{\min _{j}\left\|A_{(j)}^{-1}\right\|_{\infty}}{2} q=\infty .
\end{aligned}
$$

$$
\begin{aligned}
& \left|P_{r_{c}}(i, j)-P_{r_{d}}(i, j)\right| \\
& =\left|\frac{\sum_{j_{1}=a_{j-1}+1}^{a_{j}} \sum_{i_{1}=a_{i-1}+1}^{a_{i}} P_{\Delta_{u(i)}}\left(i_{1}, j_{1}\right) \eta_{c}\left(i_{1}\right)}{\zeta_{c}(i)}-\frac{\sum_{j_{1}=a_{j-1}+1}^{a_{j}} \sum_{i_{1}=a_{i-1}+1}^{a_{i}} P_{\Delta_{u(i)}}\left(i_{1}, j_{1}\right) \eta_{u(i)}\left(i_{1}\right)}{\zeta_{u(i)}(i)}\right| \\
& =\left|\frac{\sum_{i_{1}=a_{i-1}+1}^{a_{i}} \beta_{u(i)}\left(i_{1}, j\right) \eta_{c}\left(i_{1}\right)}{\zeta_{c}(i)}-\frac{\sum_{i_{1}=a_{i-1}+1}^{a_{i}} \beta_{u(i)}\left(i_{1}, j\right) \eta_{u(i)}\left(i_{1}\right)}{\zeta_{u(i)}(i)}\right| \\
& =\mid \frac{t_{u(i)}(i, j) \sum_{i_{1}=a_{i-1}+1}^{a_{i}} \eta_{c}\left(i_{1}\right)}{\zeta_{c}(i)}+\frac{\sum_{i_{1}=a_{i-1}+1}^{a_{i}} \epsilon_{u(i)}\left(i_{1}, j\right) \eta_{c}\left(i_{1}\right)}{\zeta_{c}(i)} \\
& -\frac{t_{u(i)}(i, j) \sum_{i_{1}=a_{i-1}+1}^{a_{i}} \eta_{u(i)}\left(i_{1}\right)}{\zeta_{u(i)}(i)}-\frac{\sum_{i_{1}=a_{i-1}+1}^{a_{i}} \epsilon_{u(i)}\left(i_{1}, j\right) \eta_{u(i)}\left(i_{1}\right)}{\zeta_{u(i)}(i)} \mid \\
& =\left|t_{u(i)}(i, j)-t_{u(i)}(i, j)+\frac{\sum_{i_{1}=a_{i-1}+1}^{a_{i}} \epsilon_{u(i)}\left(i_{1}, j\right) \eta_{c}\left(i_{1}\right)}{\zeta_{c}(i)}-\frac{\sum_{i_{1}=a_{i-1}+1}^{a_{i}} \epsilon_{u(i)}\left(i_{1}, j\right) \eta_{u(i)}\left(i_{1}\right)}{\zeta_{u(i)}(i)}\right| \\
& \leq \epsilon \frac{\sum_{i_{1}=a_{i-1}+1}^{a_{i}} \eta_{c}\left(i_{1}\right)}{\zeta_{c}(i)}+\epsilon \frac{\sum_{i_{1}=a_{i-1}+1}^{a_{i}} \eta_{u(i)}\left(i_{1}\right)}{\zeta_{u(i)}(i)} \\
& \leq 2 \epsilon \text {. }
\end{aligned}
$$




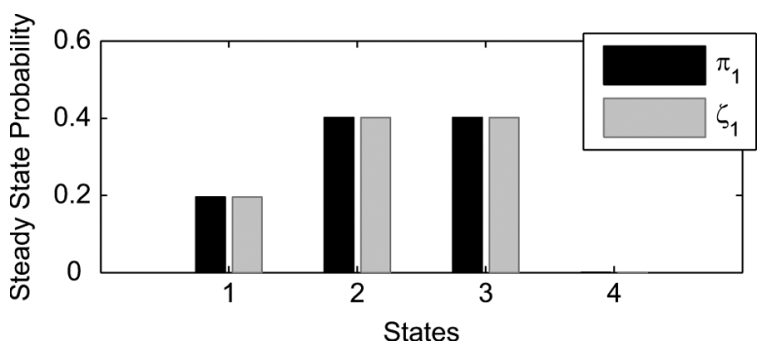

Fig. 2. Steady State Probability Distributions $\pi_{1}$ and $\zeta_{1}$.

From Theorem V.1, $|E|_{\infty} \leq 2 N \epsilon$ and if the condition number for $P_{d}$ is small, then we are sure that the outcome of the control policy designed on $P_{d}$ when applied to the fine-scale model will have coarse-scale behavior closely following $\pi_{d}$.

\section{EXAMPLE I}

We apply the reduction technique to a genetic toggle switch where there are two competing proteins $U_{1}$ and $U_{2}$, each of which inhibits the transcription of the other [37], [38]. The decay reactions $U_{1} \rightarrow \phi$ and $U_{2} \rightarrow \phi$ have propensities $c \Psi_{1}$ and $c \Psi_{2}$, respectively. The transcription of new copies of the proteins are guided by the reactions $\phi \rightarrow U_{1}$ and $\phi \rightarrow U_{2}$ with propensities $b /\left(b+\Psi_{2}\right)$ and $b /\left(b+\Psi_{1}\right)$, respectively. Based on [37], we consider $b=0.4$ and $c=0.05$ as the starting system. We truncate the maximum number of protein molecules for $U_{1}$ and $U_{2}$ to 39 resulting in a total number of $40 \times 40=1600$ possible states for the CME. Based on the propensity functions and parameter values $b=0.4$ and $c=0.05$, the state reaction matrix, $A_{1}$, is generated and the continuous Markov chain is approximated by a discrete chain using the following equation $P_{\Delta_{1}}=e^{A_{1} \Delta t}$ with a time step of $\Delta t=0.1$ seconds. The thresholds for binarization of the proteins $U_{1}$ and $U_{2}$ are selected to be $T_{1}=T_{2}=6$ molecules. ${ }^{1}$ After binarization, the two protein reduced network has $2 \times 2=4$ states. We calculated the transition probabilities of the reduced model (PBN $P_{r_{1}}$ ) using (8). In our case, the Markov chain $P_{\Delta_{1}}$ is ergodic as all the states are communicating, recurrent and aperiodic. Due to the ergodicity, $P_{\Delta_{1}}$ has a unique steady state probability distribution. The equivalence of the steady state probability distribution $\pi_{1}$ of $P_{r_{1}}$ and the collapsed steady state probability distribution $\zeta_{1}$ of $P_{\Delta_{1}}$ is shown in Fig. 2.

To study the effect of the control policy designed on the reduced network being applied to the original network, we consider a control problem similar to (5). The control input is assumed to increase the decay of the proteins by increasing the value of the parameter $c$ to 0.1 . Let $P_{\Delta_{2}}$ denote the $M \times M$ transition probabilities corresponding to the system with $c=0.1$ and $b=0.4 . P_{r_{2}}$ denotes the transition probability matrix of the PBN corresponding to $P_{\Delta_{2}}$ calculated using (8). We consider the control objective of reducing the number of steady state molecules of $U_{1}$ and $U_{2}$. In the PBN model, the control objective can be translated into increasing the steady state probability mass of state 1 (corresponding to binary state $\left[\psi_{1}, \psi_{2}\right]=[0,0]$ ). Thus, the desirable state is 1 and the undesirable states are 2 ,

\footnotetext{
${ }^{1}$ The threshold levels selected here are arbitrary but in real systems medicine application, it will be decided based on the modes of the distribution corresponding to cancerous and noncancerous states.
}

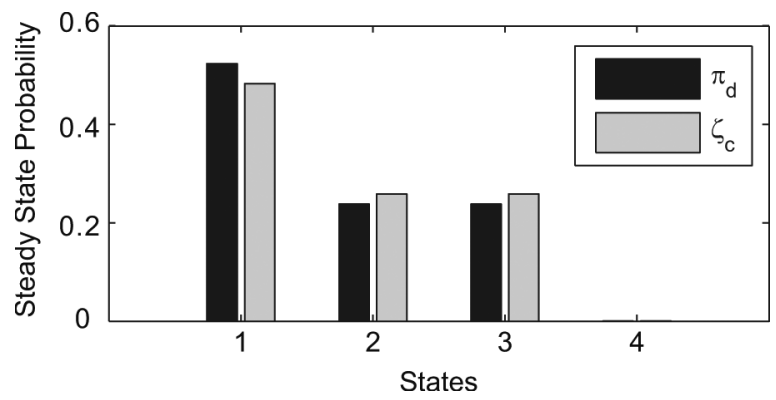

Fig. 3. Steady State Probability Distributions $\pi_{d}$ and $\zeta_{c}$.

3 , and 4 (corresponding to binary states $[0,1],[1,0]$ and $[1,1]$ respectively). The cost of control is assumed to be 0.2 and the states are assigned penalties as follows:

$$
\tilde{g}(i, u, j)= \begin{cases}0 & \text { if } u=0 \text { and } j=1 \\ .2 & \text { if } u=1 \text { and } j=1 \\ 5 & \text { if } u=0 \text { and } 2 \leq j \leq 4 \\ 5.2 & \text { if } u=1 \text { and } 2 \leq j \leq 4\end{cases}
$$

The discount factor $\alpha$ for the simulation is taken to be 0.9 . Using dynamic programming, we generated the following optimal stationary control policy: No control when in state 1 and application of control when in states 2, 3, and 4. The designed control policy is applied to the reduced PBN model and the detailed CME model. For the detailed CME model, the control policy for the states $a_{i-1}+1$ to $a_{i}$ are same as the control policy for the state $i$ of the PBN. The steady state distribution $\pi_{d}$ of the PBN and the collapsed steady state distribution $\zeta_{c}=\pi_{c}$ of the CME model after application of the control policy are shown in Fig. 3. We notice that the collapsed probability distribution $\zeta_{c}$ is not exactly same as the controlled PBN probability distribution $\pi_{d}$ but is very close to it. The steady state mass of the states with both $U_{1}$ and $U_{2}$ molecules less than 6 is higher in the controlled CME model (0.483) than in the original CME model (0.1956 as shown in Fig. 2). The $\epsilon$ for this example calculated using (13) is 0.0562 and the maximum of $\left|P_{r}(i, j)-P_{d}(i, j)\right| \forall i, j \in[1, \ldots, N]$ is 0.0061 . Thus, our bound based on Theorem V.1 is $\left|P_{r}(i, j)-P_{d}(i, j)\right|<2 \epsilon=$ $0.1124 \forall i, j \in[1, \ldots, N]$ which shows that the bound is quite loose for this example. However, for systems with lower $\epsilon$, we are guaranteed to have $\pi_{d}$ similar to $\pi_{c}$. Furthermore, $\epsilon$ can be used as an heuristic for determining threshold levels. The infinity norm of the residual $\pi_{d}\left(P_{r_{c}}-I_{N}\right)$ for the approximate solution $\pi_{d}$ for this example is $9.0922 \times 10^{-4}$ which is less than $2 \epsilon$ as proved in (18). The tightest condition number for this example was $k_{7}=62.77$ which upper bounds $\left|\pi_{d}-\pi_{c}\right|_{\infty}$ by $k_{7}\|E\|_{\infty}=62.77 \times 0.0111=0.69 .\left|\pi_{d}-\pi_{c}\right|_{\infty}$ for this example is actually 0.04 which is less than the upper bound of 0.69 . It however shows that the bound based on condition numbers is highly weak for this case.

Another parameter that can influence the difference between $\pi_{d}$ and $\pi_{c}$ is $\Delta t$ used to convert the continuous time Markov chain to discrete time Markov chain. We next observe the change in $\epsilon$ with $\Delta t$. In Fig. 4, we plotted $\epsilon$ versus $\Delta t$ at intervals of 0.5 seconds starting at 0.1 second. We notice that the value of $\epsilon$ monotonically increases at first reaching a maximum and then monotonically decreases. The minimum value of $\epsilon$ 


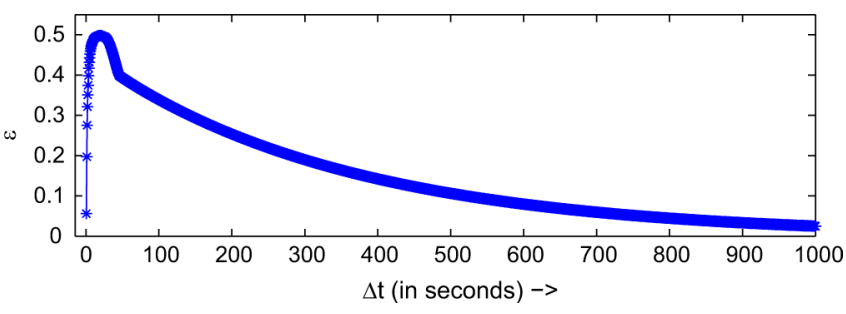

Fig. 4. Plot of $\epsilon$ versus $\Delta t$.

will be zero when $\Delta t$ is extremely large as that will signify the steady state transition probability matrix with all rows equal to the steady state probability vector. A lower $\Delta t$ to discretize the continuous time CME model will keep the value of $\epsilon$ low but a control policy designed for extremely low values of $\Delta t$ will not be implementable in practice as a drug cannot be provided or removed in extremely small time periods. Furthermore, a control policy designed using a model discretized at extremely low $\Delta t$ will require the cost of control to be much smaller. For instance, if we change $\Delta t$ of the current example from 0.1 to 0.01 , the current cost of control will produce a control policy of no action with steady state probability distribution of the controlled chain equal to $\pi_{1}$. However, if the cost of control is changed to 0.02, then we have results similar to Fig. 3 .

As the plot in Fig. 4 suggests, a high value of $\Delta t$ produces lower $\epsilon$. However, designing at high $\Delta t$ may not produce the desired control outcome as the network will not be controlled for long periods $(\Delta t)$ of time. To illustrate, let us consider that $\Delta t$ is extremely large (large enough to reach steady state behavior) and thus $P_{r_{1}}$ and $P_{r_{2}}$ will have all rows equal to $\pi_{1}$ and $\pi_{2}$ respectively. In this scenario, let us denote the controlled (stationary control policy) transition probability matrix by $P_{r_{d}}$ whose rows are either $\pi_{1}$ or $\pi_{2}$. Then,

Theorem VI.1: $\min \left(\pi_{1}(i), \pi_{2}(i)\right) \leq \pi_{r_{d}}(i) \leq$ $\max \left(\pi_{1}(i), \pi_{2}(i)\right) \forall i \in[1, \ldots, N]$.

Proof:

$$
\begin{aligned}
\pi_{r_{d}} & =\pi_{r_{d}} P_{r_{d}} \quad \forall I \in[1, \ldots, N] \\
\pi_{r_{d}}(i) & =\pi_{1}(i) \sum_{j=1, U(j) \neq 2}^{n} \pi_{r_{d}}(j)+\pi_{2}(i) \sum_{j=1, U(j) \neq 1}^{n} \pi_{r_{d}}(j) .
\end{aligned}
$$

Thus,

$$
\min \left(\pi_{1}(i), \pi_{2}(i)\right) \leq \pi_{r_{d}}(i) \leq \max \left(\pi_{1}(i), \pi_{2}(i)\right)
$$

Consequently, if our objective is to produce $\pi_{r_{d}}(i) \geq$ $\max \left(\pi_{1}(i), \pi_{2}(i)\right)$ or $\pi_{r_{d}}(i) \leq \min \left(\pi_{1}(i), \pi_{2}(i)\right)$ for some $i$, a large $\Delta t$ will not produce that.

As mentioned earlier in Section I, the computational cost involved in designing the control policy for a detailed model is enormous. However, we have a manageable 1600 states in the detailed model for this specific example allowing the generation of a control policy based on the SME model to check its performance as compared to $\zeta_{c}$. For the 1600 state SME model, we assumed a zero cost of control and the states are assigned penalties as follows:

$$
\tilde{g}(i, u, j)= \begin{cases}0 & \text { if } u=0 \text { or } 1 \text { and } 1 \leq j \leq 36 \\ 5 & \text { if } u=0 \text { or } 1 \text { and } 37 \leq j \leq 1600\end{cases}
$$

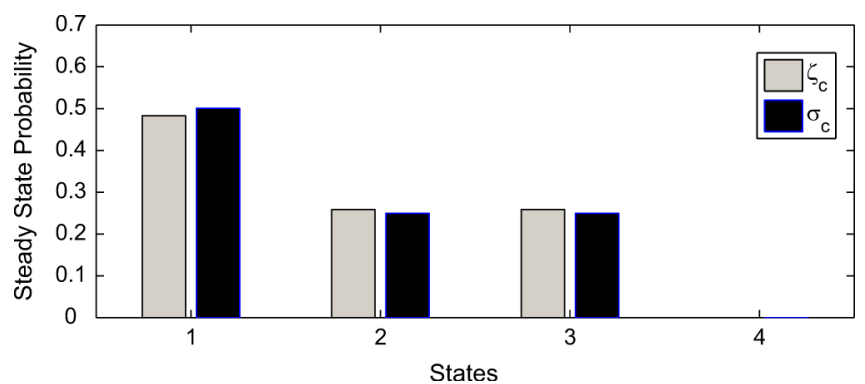

Fig. 5. Steady State Probability Distributions $\zeta_{c}$ and $\sigma_{c}$.

TABLE I

PARAMETERS OF THE StOchastic MASTER Equation MOdEL

\begin{tabular}{|c|c|c|c|c|c|}
\hline & $i=1$ & $i=2$ & $i=3$ & $i=4$ & $i=5$ \\
\hline$a_{i}$ & 0.5 & 1 & 0.15 & 0.3 & 0.3 \\
\hline$b_{i}$ & 0.12 & 0.6 & 1 & 1 & 1 \\
\hline$c_{i}$ & 0.0025 & 0.0007 & 0.0231 & 0.01 & 0.01 \\
\hline
\end{tabular}

Let $\sigma_{c}$ denotes the collapsed steady state distribution corresponding to the controlled detailed model where the control policy has been designed based on the detailed SME model. Fig. 5 shows the steady states $\zeta_{c}$ and $\sigma_{c}$. We observe that $\sigma_{c}$ outperforms $\zeta_{c}$ by having more probability in the desirable state 1 but the difference is not significant. This shows that the policy designed on the reduced PBN is performing close to the policy designed on the detailed SME with respect to the collapsed steady state.

\section{EXAMPLE II}

Our reduction procedure is also applied to a model representing the life cycle of bacteriophage- $\lambda$ [37], [38] which involves five proteins $U_{1}, U_{2}, \ldots, U_{5}$. The decay reactions $U_{i} \rightarrow \phi$ for $i=1,2, . ., 5$ are with propensities $c_{i} \Psi_{i}$ for $i=1,2, . ., 5$. The generation reactions with their respective propensities are as follows:

1) $\phi \rightarrow U_{1}$ with propensity $a_{1} b_{1} /\left(b_{1}+\Psi_{2}\right)$;

2) $\phi \rightarrow U_{2}$ with propensity $\left(a_{2}+\Psi_{5}\right) b_{2} /\left(b_{2}+\Psi_{1}\right)$;

3) $\phi \rightarrow U_{3}$ with propensity $a_{3} b_{3} \Psi_{2} /\left(b_{3} \Psi_{2}+1\right)$;

4) $\phi \rightarrow U_{4}$ with propensity $a_{4} b_{4} \Psi_{3} /\left(b_{4} \Psi_{3}+1\right)$;

5) $\phi \rightarrow U_{5}$ with propensity $a_{5} b_{5} \Psi_{3} /\left(b_{5} \Psi_{3}+1\right)$.

The generation reactions for the proteins originate from the mechanism of transitions between two phases, lysogenic and lytic, present typically in the life of a bacteriophage- $\lambda$. Alterations from one phase to another are regulated by a toggle switch of two competing proteins $U_{1}$ and $U_{2}$, which inhibit each other's transcription. In the lysogenic phase $U_{2}$ is abundant while suppressing the production of $U_{1}$ via the reaction 1 . The generation of $U_{2}$ via reaction 2, on the other hand, depends on the amount of $U_{5}$. Under a stressed situation $U_{5}$ decreases as the phage transitions into the lytic stage. The parameters $a_{i}, b_{i}$, $c_{i}$ are taken from [37] as shown in Table I.

The maximum number of protein molecules for $U_{1}, U_{2}, U_{3}$, $U_{4}$, and $U_{5}$ are truncated to be at $3,24,3,3$, and 2, respectively so that the total number of possible states for the CME is $4 \times 25 \times 4 \times 4 \times 3=4800$. Using the above propensity functions and values for parameters $a_{i}, b_{i}, c_{i}$ the state reaction 


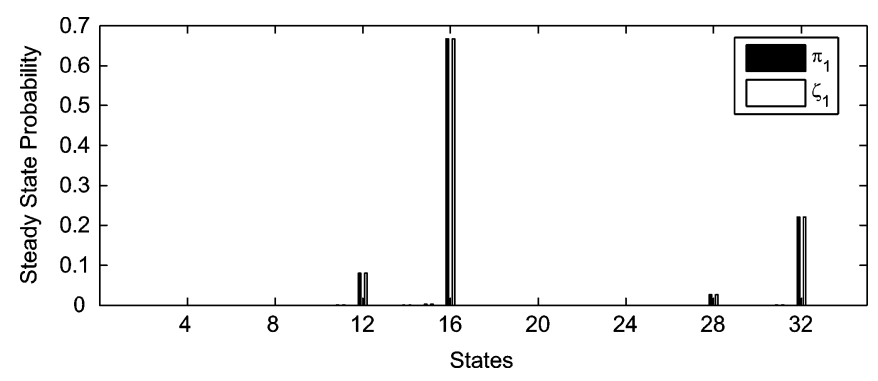

Fig. 6. Steady State Probability Distributions $\pi_{1}$ and $\zeta_{1}$.

matrix, $A_{1}$ is computed and the continuous Markov chain is approximated by a discrete chain using equation $P_{\Delta 1}=e^{A_{1} \Delta t}$ with a time step of $\Delta t=0.1$ seconds. The binarization of the proteins is achieved using thresholds $2,8,2,1$, and 1 , respectively. After binarization, the five protein reduced network has $2^{5}=32$ states. The transition probabilities of the reduced model (PBN $P_{r_{1}}$ ) are calculated using (8). Since all the states are communicating, recurrent and aperiodic, the Markov chain $P_{\Delta 1}$ is ergodic and hence $P_{\Delta 1}$ has a unique steady state probability distribution. The equivalence of the steady state probability distribution $\pi_{1}$ of $P_{r 1}$ and the collapsed steady state probability distribution $\zeta_{1}$ of $P_{\Delta 1}$ is shown in Fig. 6.

Next, we proceed to study the effect of the control policy designed on the reduced network being applied to the original network. We consider the control objective of reducing the steady state masses of the high states in Fig. 6. Thus, the undesirable states are 16, 32 (corresponding to binary states $[0,1,1,1,1]$, and $[1,1,1,1,1]$, respectively), and all other states are desirable. Similar to the two gene case, the cost of control is assumed to be 0.2 and the discount factor $\alpha$ is taken to be 0.9 . Using dynamic programming, we generated the following optimal stationary control policy: application of control when in states 16 and 32 and no control when in all other states. The designed control policy is applied to both the reduced PBN model and the detailed CME model. The control policy for the states $a_{i-1}+1$ to $a_{i}$ in the detailed CME model are same as the control policy for the state $i$ of the PBN. The steady state distribution $\pi_{d}$ of the PBN and the collapsed steady state distribution $\zeta_{c}=\pi_{c}$ of the CME model after application of the control policy are shown in Fig. 7. Similar to the two gene case, we observe that the collapsed probability distribution $\zeta_{c}$ and the controlled PBN probability distribution $\pi_{d}$ are very close to each other, though not exactly the same. We also notice that the steady state mass of the undesirable states is lower in Fig. 7 as compared to Fig. 6 while many of the desirable states have increased steady state masses, indicating that the control is effective.

The $\epsilon$ for this example is 0.1487 . Thus, according to Theorem V.1, $\left|P_{r}(i, j)-P_{d}(i, j)\right| \forall i, j \in[1, \ldots, N]$ should be $\leq 2 \epsilon=0.2974$. For this example, the maximum of $\mid P_{r}(i, j)-$ $P_{d}(i, j) \mid \forall i, j \in[1, \ldots, N]$ is 0.0335 which is less than $2 \epsilon=$ 0.29 . The infinity norm of $\pi_{c}-\pi_{d}$ for this example is 0.0365 . The tightest condition number was $k_{3}=120$ which upper bounds $\left|\pi_{d}-\pi_{c}\right|_{\infty}$ by $k_{3}\|E\|_{\infty}=120 \times 0.0691=8.292$. This shows that the bound based on the condition number is not providing any new information for this specific example. The infinity norm of the residual $\pi_{d}\left(P_{r_{c}}-I_{N}\right)$ for the approximate

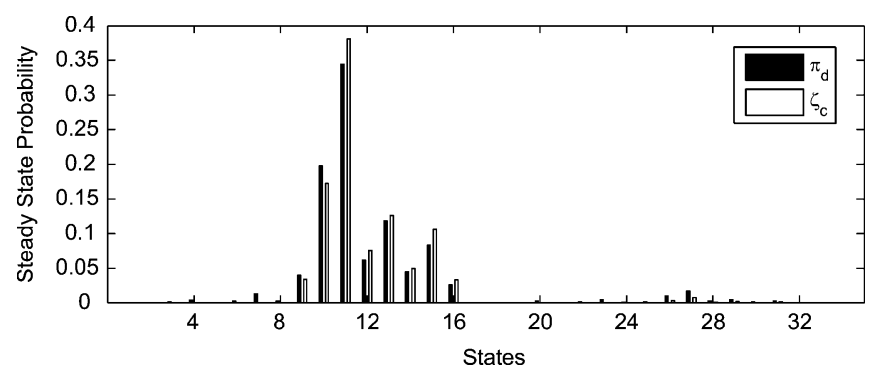

Fig. 7. Steady State Probability Distributions $\pi_{d}$ and $\zeta_{c}$.

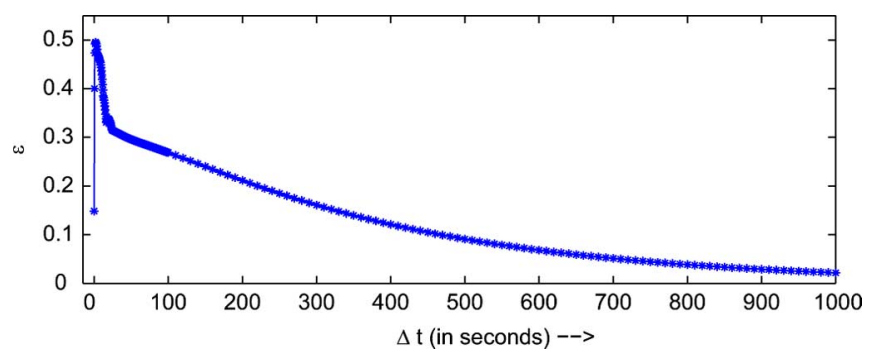

Fig. 8. Plot of $\epsilon$ versus $\Delta t$.

solution $\pi_{d}$ for this example is 0.0013 which is less than $2 \epsilon$ as proved in (19).

To study the change in value of $\epsilon$ relative to $\Delta t$, in Fig. 8 we plotted $\epsilon$ versus $\Delta t$ at intervals of 0.5 seconds starting at 0.1 second until 100 seconds. Then the interval was increased to 10 seconds. We notice that the value of $\epsilon$ increases at first reaching a maximum and then after two small oscillations decreases monotonically.

\section{CONCLUSION}

In this paper, we presented a reduction mapping from fine scale stochastic models represented by CMEs to coarse-scale models represented by PBNs. The mapping preserves the collapsed steady state distribution of the CME model. We formulated a parameter $\epsilon$ whose low value provides a sufficient condition for similarity of the steady state probability distributions of the controlled PBN $\pi_{d}$ and the collapsed steady state probability distribution of the controlled CME $\pi_{c}$. Bounds on the difference in the steady state probability distributions of $\pi_{d}$ and $\pi_{c}$ in terms of $\epsilon$ were derived. The effect of the control policy designed using the reduced network when applied to the fine scale model was evaluated through two biological examples. The simulations showed that the result of the control policy application on the fine-scale model was not far away from the result observed with the PBN model.

\section{REFERENCES}

[1] A. Arkin, J. Ross, and H. H. McAdams, "Stochastic kinetic analysis of developmental pathway bifurcation in phage-infected escherichia coli cells," Genetics, vol. 149, pp. 1633-1648, 1998.

[2] H. H. McAdams and A. Arkin, "Stochastic mechanisms in gene expression,” Proc. Nat. Acad. Sci, vol. 94, pp. 814-819, 1997.

[3] D. T. Gillespie, "A rigorous derivation of the chemical master equation," Physica A, vol. 188, pp. 404-425, 1992.

[4] R. Pal, A. Datta, and E. R. Dougherty, "Optimal infinite horizon control for probabilistic Boolean networks," IEEE Trans. Signal Process., vol. 54, no. 6, pp. 2375-2387, Jun. 2006. 
[5] M. Bittner, P. Meltzer, Y. Chen, Y. Jiang, E. Seftor, M. Hendrix, M. Radmacher, R. Simon, Z. Yakhini, A. Ben-Dor, N. Sampas, E. R. Dougherty, E. Wang, F. Marincola, C. Gooden, J. Lueders, A. Glatfelter, P. Pollock, J. Carpten, E. Gillanders, D. Leja, K. Dietrich, C. Beaudry, M. Berens, D. Alberts, and V. Sondak, "Molecular classification of cutaneous malignant melanoma by gene expression profiling," Nature, vol. 406, pp. 536-540, 2000.

[6] A. Datta, R. Pal, A. Choudhary, and E. R. Dougherty, "Control approaches for probabilistic gene regulatory networks," IEEE Signal Process. Mag., vol. 24, pp. 54-63, 2007.

[7] H. D. Jong, "Modeling and simulation of genetic regulatory systems: A literature review," J. Comput. Biol., vol. 9, pp. 67-103, 2001.

[8] D. P. Bertsekas, Dynamic Programming and Optimal Control, 2nd ed. Nashua, NH: Athena Scientific, 2001.

[9] Z. Szallasi, J. Stelling, and V. Periwal, System Modeling in Cell Biology from Concepts to Nuts and Bolts. Cambridge, MA: MIT Press, 2006.

[10] X. Cai and X. Wang, "Stochastic modeling and simulation of gene networks : A review of the state-of-the-art research on stochastic simulations," IEEE Signal Process. Mag., vol. 24, pp. 27-36, 2007.

[11] J. M. Bower and H. Bolouri, Computational Modeling of Genetic and Biochemical Networks, 1 ed. Boston, MA: MIT Press, 2001.

[12] R. Heinrich and S. Schuster, The Regulation of Cellular Systems. New York: Chapman \& Hall, 1996.

[13] E. O. Voit, Computational Analysis of Biochemical Systems: A Practical Guide for Biochemists and Molecular Biologists. Cambridge, U.K.: Cambridge Univ. Press, 2000.

[14] P. Smolen, D. Baxter, and J. Byrne, "Mathematical modeling of gene networks," Neuron, vol. 26, pp. 567-580, 2000.

[15] L. Glass and S. A. Kauffman, "The logical analysis of continuous, nonlinear biochemical control networks," J. Theoretic. Biol., vol. 39, pp. 103-129, 1973.

[16] T. Mestl, E. Plahte, and S. W. Omholt, "A mathematical framework for describing and analysing gene regulatory networks," J. Theoretic. Biol., vol. 176, pp. 291-300, 1995.

[17] M. Gibson and E. Mjolsness, Modeling the Activity of Single Genes in Computational Methods in Molecular Biology. Cambridge, MA: MIT Press, 2001.

[18] H. H. McAdams and A. Arkin, "It's a noisy business! genetic regulation at the nanomolar scale," Trends Genet., vol. 15, no. 2, pp. 65-69, 1999.

[19] G. Nicolis and I. Prigogine, Self-Organization in Nonequilibrium Systems: From Dissipative Structures to Order Through Fluctuations. New York: Wiley, 1977.

[20] Z. Szallasi, "Genetic network analysis in light of massively parallel biological data acquisition," in Proc. Pac. Symp. Biocomput. (PSB), 1999, pp. 5-16.

[21] D. T. Gillespie, "Exact stochastic simulation of coupled chemical reactions," J. Phys. Chem., vol. 81, pp. 2340-2361, 1977.

[22] I. Golding, J. Paulsson, S. M Zawilski, and E. C Cox, "Real-time kinetics of gene activity in individual bacteria," Cell, vol. 123, pp. 1025-1036, 2005.

[23] B. Munsky and M. Khammash, "The finite state projection algorithm for the solution of the chemical master equation," J. Chem. Phys., vol. 124, no. 044104, 2006.

[24] S. A. Kauffman, The Origins of Order: Self-Organization and Selection in Evolution. New York: Oxford Univ. Press, 1993.

[25] I. Shmulevich, E. R. Dougherty, S. Kim, and W. Zhang, "Probabilistic Boolean networks: A rule-based uncertainty model for gene regulatory networks," Bioinformatics, vol. 18, pp. 261-274, 2002.

[26] N. Friedman, M. Linial, I. Nachman, and D. Pe'er, "Bayesian networks to analyze expression data," in Proc. 4th Annu. Int. Conf. Computational Molecular Biology, 2000, pp. 127-135.

[27] H. Lahdesmaki, S. Hautaniemi, I. Shmulevich, and O. Yli-Harja, "Relationships between probabilistic Boolean networks and dynamic Bayesian networks as models of gene regulatory networks," Signal Process., vol. 86, pp. 814-834, 2006.
[28] A. A. Julius, A. Halasz, M. S. Sakar, H. Rubin, V. Kumar, and G. J. Pappas, "Stochastic modeling and control of biological systems: The lactose regulation system of escherichia coli," IEEE Trans. Autom. Control, vol. 53, pp. 51-65, 2008.

[29] J. W. Clark, Harrison's Manual of Oncology. New York: McGrawHill Professional, 2007, ch. 1.10, Molecular Targeted Drugs, pp. 67-75.

[30] "Roche applied science, Integrated solutions for gene knockdown," Biochemica no. 4, 2004.

[31] I. Shmulevich, E. R. Dougherty, and W. Zhang, "Gene perturbation and intervention in probabilistic Boolean networks," Bioinformatics, vol. 18, pp. 1319-1331, 2002.

[32] A. Datta, A. Choudhary, M. L. Bittner, and E. R. Dougherty, "External control in Markovian genetic regulatory networks," Mach. Learn., vol. 52, pp. 169-191, 2003.

[33] R. Pal, A. Datta, M. L. Bittner, and E. R. Dougherty, "Intervention in context-sensitive probabilistic Boolean networks," Bioinformatics, vol. 21, pp. 1211-1218, 2005.

[34] R. Pal and S. Bhattacharya, "Steady-state preserving reduction for genetic regulatory network models," in Proc. IEEE Int. Symp. ComputerBased Medical Systems (CBMS), 2009 [Online]. Available: http://dx. doi.org/10.1109/CBMS.2009.5255246, Article DOI: 10.1109/CBMS 2009.5255246

[35] T. Kailath, Linear Systems. Englewood Cliffs, NJ: Prentice-Hall, 1979.

[36] G. E. Cho and C. D. Meyer, "Comparison of perturbation bounds for the stationary distribution of a Markov chain," Linear Algebra Appl., vol. 335, pp. 137-150, 2001.

[37] M. Hegland, C. Burden, L. Santoso, S. MacNamara, and H. Booth, "A solver for the stochastic master equation applied to gene regulatory networks," J. Comput. Appl. Math., vol. 205, no. 2, pp. 708-724, 2007.

[38] T. Jahnke and W. Huisinga, "A dynamical low-rank approach to the chemical master equation," Bull. Math. Biol., vol. 70, pp. 2283-2302, 2008.

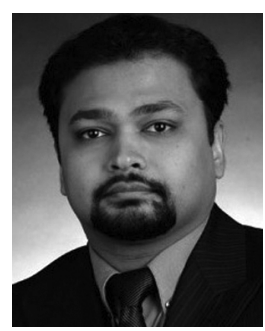

Ranadip Pal (S'05-M'07) received the B.Tech degree in electronics and electrical communication engineering from the Indian Institute of Technology, Kharagpur, India, in 2002 and the M.S. and Ph.D. degrees in electrical engineering from Texas A\&M University, College Station, in 2004 and 2007, respectively.

In August 2007, he joined the Department of Electrical and Computer Engineering at Texas Tech University, Lubbock, as an Assistant Professor. His research areas are computational biology, genomic signal processing, and control of genetic regulatory networks.

Dr. Pal is the recipient of 2010 NSF CAREER Award. While at Texas A\&M, he was the recipient of the Ebensberger/Fouraker Fellowship, a Distinguished Graduate Student Masters Research Award, and a National Instruments Fellowship. He was also an Indian National Math Olympiad Awardee and was ranked first in the Regional Math Olympiad, West Bengal, India.

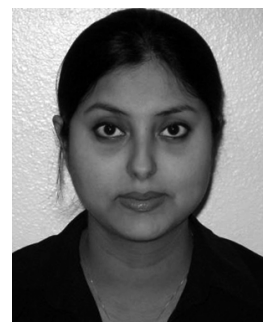

Sonal Bhattacharya received the B.Sc. and M.Sc. degrees in physics from Calcutta University, Calcutta, India, in 1998 and 2001, respectively, and the post-M.S. diploma in physics from Saha Institute of Nuclear Physics, Calcutta, India, in 2002. She is currently working towards the Ph.D. degree in electrical and computer engineering at Texas Tech University, Lubbock. Her research areas are genomic signal processing, control of genetic regulatory networks, image processing, solid-state physics, and fluid mechanics. 